\title{
Photo-recovery of Nanoparticles from an Organic Solvent
}

\section{supporting material}

\author{
Alireza Salabat ${ }^{\star}$, Julian Eastoe*, Ana Vesperinas, Rico F. Tabor, Kevin J. Mutch \\ School of Chemistry, University of Bristol, Bristol, UK BS8 1TS \\ - Chemistry Department, Arak University, Arak, Iran
}

\section{TEM}

TEM imaging of silica nanoparticles stabilized by C6PAS/AOT surfactant mixtures in toluene was carried out using a JEOL 1200EX electron microscope at $120 \mathrm{kV}$. A representative image is given in Figure S1, the scale bar represents $200 \mathrm{~nm}$.

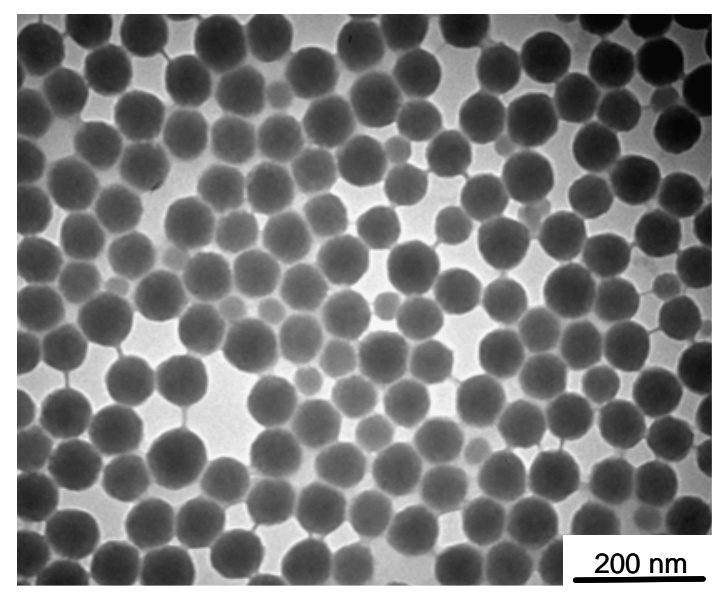

Fig.S1. TEM image of silica nanoparticles coated by an C6PAS/AOT surfactant mixture.

\section{Apparent diameter and apparent diffusion coefficient}

Apparent diameters and apparent diffusion coefficients of stabilized silica nanoparticles, measured by dynamic light scattering are reported in Figure S2 and Table 1 of the manuscript. These measurements were performed for $\mathrm{X}_{\mathrm{C} 6 \mathrm{PAS}}=10 \%$, [surfactant $]_{\mathrm{tot}}=0.10 \mathrm{~mol} \mathrm{dm}^{-3}$, one hour after irradiation. With increasing irradiation time, the apparent diameter increases and the apparent diffusion coefficient decreases. 


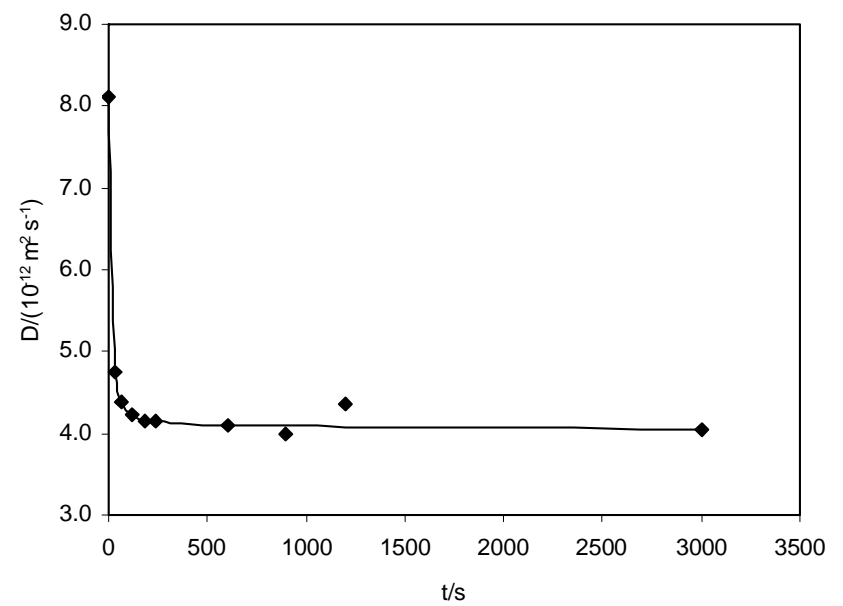

Figure S2. Variation of apparent diffusion coefficient with UV irradiation time of silica NP organosols stabilized by C6PAS/AOT mixtures in toluene. $\mathrm{X}_{\mathrm{C} 6 \mathrm{PAS}} 10 \%$, $[\text { surfactant }]_{\text {tot }}=0.10 \mathrm{~mol} \mathrm{dm}{ }^{-3}$. DLS measurements were taken 1 hour after irradiation.

\section{Flocculation of silica particles}

The irradiated solutions began to show precipitation if left overnight. This is a process which occurs slowly and takes about 3 or 4 days for complete precipitation. Figure S1 shows precipitated silica from the irradiated solution after one day of standing.

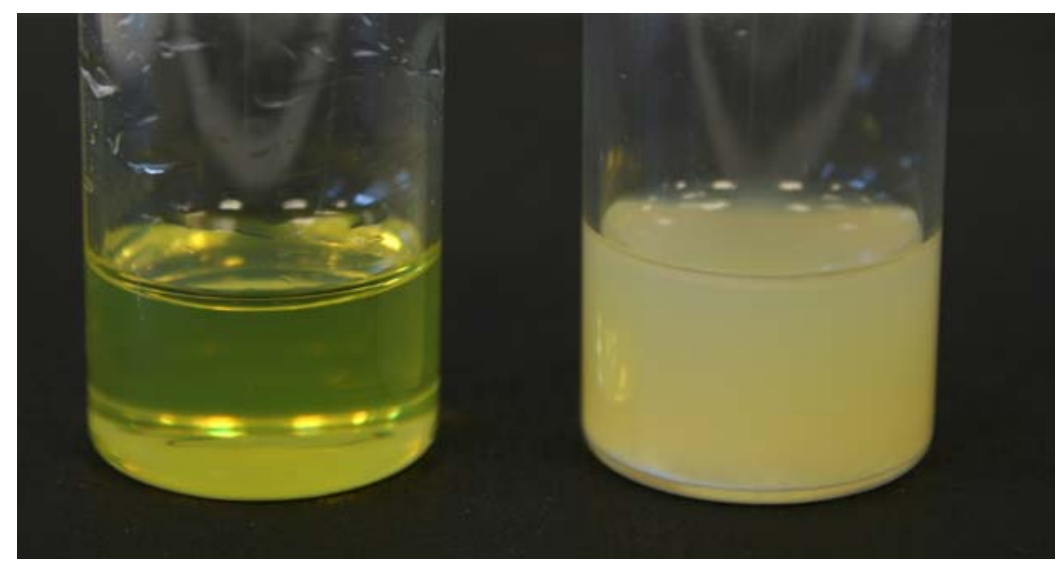

Fig. S3. Silica nanoparticles stabilized by C6PAS/AOT mixtures in toluene: $\mathrm{X}_{\text {C6PAS }}$ $(\%)=10$, [surfactant $]_{\mathrm{tot}}=0.10 \mathrm{~mol} \mathrm{\textrm {dm } ^ { - 3 }}$. Before irradiation (left); after $50 \mathrm{~min}$ irradiation and stored for one day (right). 